Article

\title{
Implementing Mobile Games into Care Services-Service Models for Finnish and Chinese Elderly Care
}

\author{
Sari Merilampi ${ }^{1, *(\mathbb{D}}$, Antti Koivisto ${ }^{1}$, Mirka Leino ${ }^{1}$, Nuno Pombo ${ }^{2}$, Virginie Felizardo ${ }^{2}$, \\ Jue $\mathrm{Lu}^{3}$, Anja Poberznik ${ }^{1}$ and Johanna Virkki ${ }^{4}$ (D) \\ 1 Faculty of Technology, Satakunta University of Applied Sciences, 28130 Pori, Finland; \\ antti.koivisto@samk.fi (A.K.); mirka.leino@samk.fi (M.L.); anja.poberznik@samk.fi (A.P.) \\ 2 Department of Informatics, Instituto de Telecomunicações (IT), University of Beira Interior, 6201-001 Covilhã, \\ Portugal; pombo.ubi@gmail.com (N.P.); virginie@it.ubi.pt (V.F.) \\ 3 School of Nursing, Changzhou University, Changzhou 213164, China; jue.lu@samk.fi \\ 4 Faculty of Medicine and Health Technology, Tampere University, 33720 Tampere, Finland; \\ johanna.virkki@tuni.fi \\ * Correspondence: sari.merilampi@samk.fi
}

Received: 30 November 2018; Accepted: 5 February 2019; Published: 8 February 2019

check for updates

\begin{abstract}
The purpose of this paper was to create service models for cognitively stimulating mobile games and incorporate them into Finnish and Chinese elderly care. The implementation involved the use of two different mobile games as part of the everyday lives of older adults in care homes in Finland (3 months) and China (6 months). Although a large number of publications examine serious games in elderly care, there are rather few publications related to the practical implementation within the elderly care processes. In general, rehabilitation orientated games should incorporate entertainment (motivation) and relevant therapeutic content (rehabilitation) in order to be effective. Regardless of the game design, successful implementation of the games in elderly care is paramount to benefit the end user. In this paper, two mobile games were investigated as a case study. To investigate the therapeutic content of the games, the game outcomes (game scores and time stamps) were automatically recorded to facilitate analysis of the participant's progress during the trial. To investigate motivation, user feedback was collected through observation of the game trials and by interviewing the nursing staff and the participants (test group). The gaming service implementation was designed in collaboration with the nursing staff and researchers, according to an experimentation-driven approach, in which the service model ideas were tested by the professionals before piloting. In both countries, the players and the nursing staff found the games showed potential as self-managed rehabilitation tools. Other significant effects of gameplay were enhanced recreation and self-managed activity level. Despite cultural differences, the gaming experience was amazingly similar and improvements in game scores were also observed during the trial in both countries. The biggest difference between the pilots was the implementation process, which led to the development of two different service models that are reported in this paper. In Finland, the games were embedded into the care practices and the nursing staff were responsible for the piloting. In China, the games were independent of the care process and an external service provider (the researcher) managed the piloting. The findings imply that service design in different cultures should be carefully considered when implementing new digital services.
\end{abstract}

Keywords: cultural comparison; serious games; service design; older adults; recreation; self-managed rehabilitation 


\section{Introduction}

Due to rapidly developing technologies and lack of health care resources, novel technology-assisted care services are under development. Serious games (games combining entertainment and useful content, such as educational or rehabilitation elements) are one promising solution when it comes to motivating people for self-rehabilitation and self-care [1,2]. Serious games have demonstrated transient effects and benefits towards elderly well-being. Games have been developed for both physical [3-7] and mental/cognitive well-being [8-11]. Although serious games can be a valuable option for prevention and rehabilitation, further research is needed to evaluate which settings support long-term motivation and engagement of older people in serious games [2].

Another rather rarely researched field is the actual implementation of games into an elderly care context, from a service development point of view. Other kinds of information and communication technology (ICT)-related health services (eHealth, telehealth, mHealth) are researched more commonly [12,13]. The concept of this study could be categorized under mHealth. Typical mHealth services include data collection and communication by mobile devices via the Internet, but sensors/measurement devices may also be included. In addition, mHealth services typically include remote consultation/action or feedback from a service provider (i.e., a health care professional). For example, questionnaires or self-measurements are sent to the health care professional before/during/after a care process, which makes the care more efficient and more accessible (especially useful in rural areas) [12,13].

In this study, the researchers utilized the basic concept of mHealth, combining service provider and electronic data gathering with mobile memory games, and formalized service models for implementing mobile memory games in elderly care. The models were developed through benchmarking the basic mHealth concept and refining it through experiments and discussion with health care professionals. The importance of developing service models is obvious, as no technology will start working on its own. Instead, the implementation of technology should be carefully considered and planned, as it will influence the care process. Implementation should involve analyses into how the technology will affect the existing processes and services, and if there is potential to create entirely new services based on the technology. Technology requires support services (teaching use, explaining benefits, "help desk" etc.), but also value-adding services. One major target group of digital healthcare and welfare services are the elderly. Despite different user needs and expectations, if compared to regular mobile device users, older adults are interested in and capable of handling their devices and associated applications [14-16]. The aim of the study was to investigate and pilot an implementation process for mobile memory games, for use in elder care in Finland and China (the service design being the main viewpoint). We investigated the user and care personnel experiences, since successful implementation required positive attitudes and experiences. Further, we investigated the progress of the elderly players' game scores, to analyse the well-being related benefits of the piloted gaming service. All these components (motivation, benefits, and successful implementation) needed to work together in order to maximise the benefit of serious gaming.

The games investigated in this study were originally developed for the Finnish elderly and tested in gaming trials in Finnish elderly care homes [17]. The results were extremely positive, demonstrating positive user experiences, increased social interaction, and positive feelings towards rehabilitation effectiveness. Thereafter, some initial tests in Chinese elderly care homes were performed. These results have also been encouraging, showing an interest in gaming and positive user experiences among the participants $[15,16]$. Based on the pilots performed in two different countries, two different service models were designed according to the implementation of the gaming trials. This study provides a concrete case study of a welfare technology implementation process, in which serious games targeted for elderly rehabilitation are piloted in Finnish and Chinese elderly care. 


\section{Research Methods and Test Set-Up}

The two simple mobile games used in this study were originally developed in Finland, by a multidisciplinary team including game designers, programmers, usability experts and memory rehabilitation professionals, as well as elderly test users. As the games are described in more detail in another article [17], only a brief description is given in this article to help the reader to understand the results.

The first game, Cat vs. Mouse (Figure 1), combines physical movement and cognitive stimuli. The idea was based on previous studies which implied physical exercise and game play have positive effects on elderly [18-20]. The main aim of the game is to control the mouse character to catch cheese chunks by tilting an Android mobile device. The game contains a time limit (one minute) in which the player tries to get as many cheese chunks as possible. After getting a cheese chunk, the player gets points and another cheese chunk appears in different location. After getting five cheese chunks, an opponent (a cat) appears and starts to follow the player (the mouse). New cats appear after five more cheese chunks, while also making the first cat faster. The game ends after the mouse hits a cat or the time expires. There is also an impenetrable obstacle in the game to add difficulty.

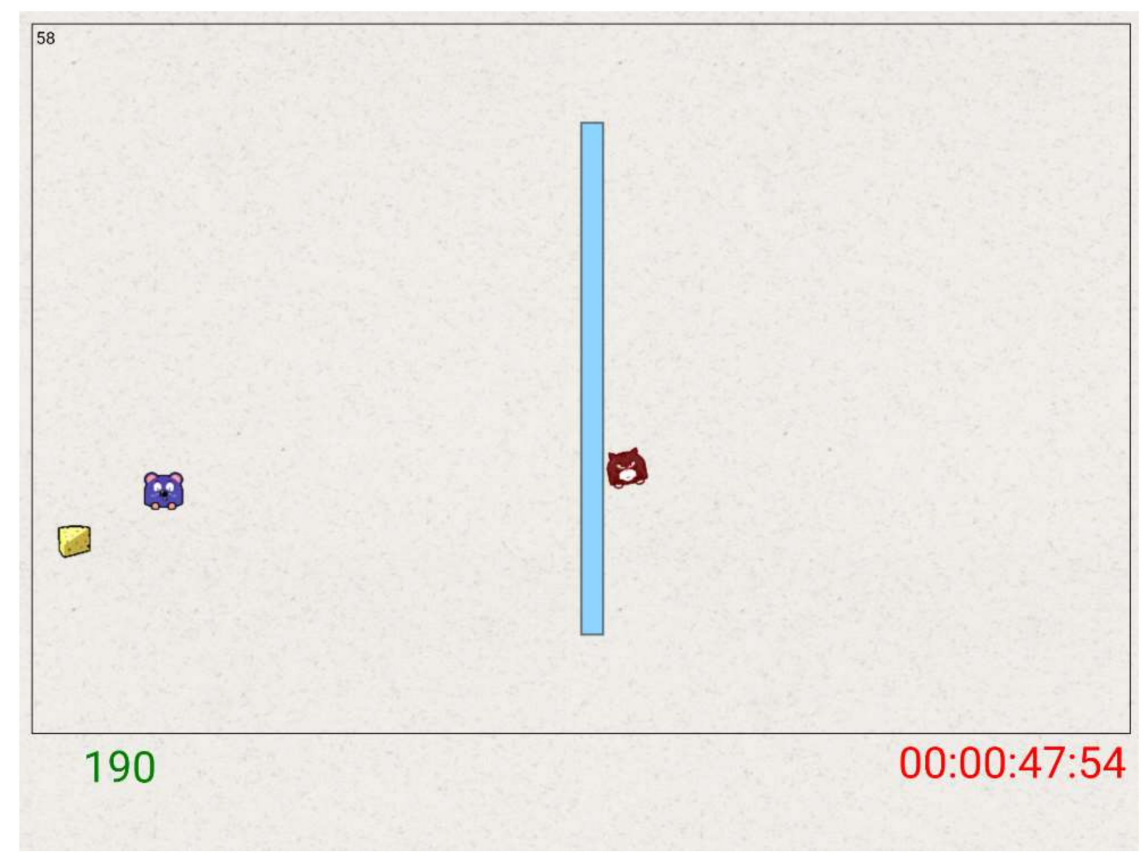

Figure 1. Game screen in Cat vs. Mouse: The blue mouse is controlled by titling the tablet. The idea is to collect cheese chunks whilst avoiding the cats.

The second game used in this study is based on the Trail Making Test (TMT) which consists of 25 numbered circles (from 1 to 25 ) in random order on a sheet of paper. The aim is to draw lines to connect the numbers in ascending order as quickly as possible, without lifting the pen or pencil from the paper. The time taken to finish this task is measured. Longer times indicate greater cognitive impairment [21-23]. The game version (Figure 2) mimics the test, but the numbers are on a tablet screen and the player must tap the numbers in ascending order. Correctly selected circle turns transparent, and incorrect taps are indicated by the incorrectly selected circle flashing red. The game ends when all circles are tapped in the correct order, or after four errors. The difficulty level can be changed in the game menu by adding or removing digits on screen. 


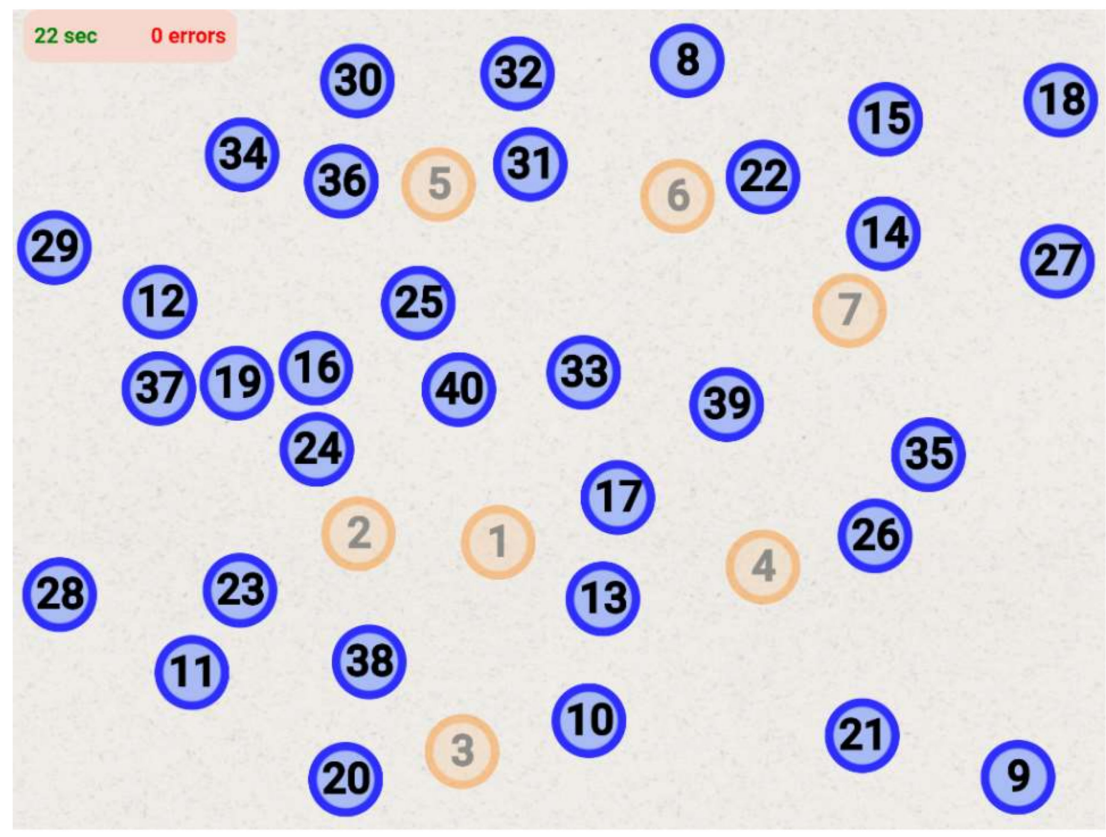

Figure 2. A modified electronic version of the Trail Making Test (TMT): The player taps the circles in ascending order as quickly as possible.

The purpose of this study was to develop service models for mobile memory games, used in care services in Finland and China. To be able to achieve this goal, we needed to justify the suitability of the games in this context. This led to research questions one and two, discussed in the Sections 3.1 and 3.2. After justifying the suitability of the games, we wanted to develop the actual service models for implementing the games, and investigate the effect of culture on the service models and suitability of the games. This led to the main research question (research question three) discussed in Section 3.3.

\subsection{User Experiences}

In order for any serious game to have a meaningful benefit, such as the mobile memory games in this study, it must be motivating enough to engage players. This requires the usability of the game to be matched to the users' capabilities. In this study, we applied Jesse Garrett's user experience frame [24] as a guideline to answer the first research question (RQ), RQ1: Are the games suitable, and is the gaming experience perceived to be meaningful (by the elderly and the nursing personnel)?

In Garrett's usability frame, usability considers a much wider scope than being "easy-to-use". In particular, the focus of this research is associated with the experienced meaningfulness of the games, as it has huge impact on motivation. The research methods related to user experiences are: 1. Questionnaires to nursing staff and elderly players, and 2. Observations during game trials. The research was themed according to Garrett's usability frame: Visual design, information and interaction design, functional specifications and user needs.

\subsection{Gaming Scores}

Although the main focus of this study was not evaluating the therapeutic effect of the games, the effect of frequent gaming on game scores was automatically recorded. This facilitated evaluation of the game scores and whether they improved over the gaming period, as the game play requires cognitive activity. Thus, we formulated the second $R Q, R Q 2$ : Do the game scores improve through frequent gaming? If there is no improvement, this might indicate either inappropriate game design or gaming service model, considering the user group. Improved game scores, on the other hand, would further justify the suitability of the gaming services and encourage further studies regarding the health effects. 


\subsection{Gaming Service Models}

The main research focus of the study is RQ3: Is there any difference between cultures considering (a) the way the gaming could be implemented, and (b) considering RQ1 and 2, would these factors affect the service models? In order to answer RQ3, the gaming service implementation was designed in collaboration with nursing staff and researchers, using an experimentation-driven approach, in which the service model ideas were tested by the professionals before implementation. As effective rehabilitation is typically a long-term process requiring commitment and persistence in order to attain and maintain desired abilities $[19,25,26]$, the gaming periods were originally designed to be 3 months. In addition, the relatively long test period was selected to test participants' motivation after the possible charm or novelty had worn off. The game scores of all test group members were recorded to investigate the gaming activity (motivation) and progress (rehabilitation effects; i.e., game scores).

The authors of the Finnish study presented the Finnish game trial [17], in which the same serious games were played five minutes of each game daily for three months, after which the gaming progress was investigated. The participants were 10 elderly individuals (male and female), with an average age of 90, living in an elderly care home in Pori, Finland. The nursing personnel were in charge of the trials, taking care of the tablets, giving advice and offering the opportunity to play each day.

The original plan was to repeat the same in China; however, according to the wishes of the nursing staff, an external service provider controlled the gaming trial. The protocol was adjusted accordingly to this model. A major challenge related to the open-door nature of the care home, making the staff afraid that somebody would steal the tablets. Trust in Chinese society is linked to the Confucian virtues, as well as the concepts of guanxi and mianzi that shape human relationships, social structures and social orientation of the Chinese culture [27]. Guanxi is a concept referring to the tight social networks that shape Chinese society. Mianzi, or the concept of "face", refers to the respect, pride and dignity of the person and is related to the person's status in the society. Along with Confucianism, a traditional hierarchical family system and centralized government system affect the present-day characteristics of Chinese culture, in which masculine domination, hierarchy, bureaucracy, respect for age, importance of interpersonal relationships and saving "face" reflect themselves in the Chinese society [28]. All these aspects also influence building trust in Chinese society. Interpersonal trust in Chinese society emerges through shared kinship and close-knit social circles and it is most often applied to others through family connections [29]. Residents of the elderly home in China, the nursing personnel, visitors and potential outsiders are not from the same guanxi (as they are all strangers initially), which might explain the lack of trust and fear of theft. It is not common that a stranger would be trusted automatically. It has also been proposed that whereas Westerners start from the basis of ambivalent or neutral trust (i.e., zero trust) when meeting strangers, the Chinese start from a position of distrust [29]. Finnish culture, on the other hand, is different. Finnish people are generally described as naturally cautious in trusting strangers, but their trust in people in general is very high. They also have high standards for stamina, workmanship, reliability and safety $[27,30]$.

Thus, due to the cultural difference in nursing home practices, two completely different types of gaming implementations were required. Eventually, the games were played in China on a weekly basis (gaming events on Saturdays) in an elderly home in Changzhou, China. The duration of the game trial in China was scheduled for 6 months, in order to obtain an adequate amount of data to detect possible tendencies in gaming processes and score developments. During the study, the researcher brought the device on-site and the 10 participants (male and female, with an average age of 84 years) played the games, if they were willing. During the study, the games were played in a semi-controlled environment, in which care staff were present and a Chinese-speaking researcher was there to observe and help, if needed. 


\section{Results and Discussion}

\subsection{User Experiences}

In this study, user experience and usability are defined by ISO 9241-210 [16] as, "the person's perceptions and responses that result from the use or anticipated use of a product, system or service". Thus, user experience includes all the users' emotions, beliefs, preferences, perceptions, physical and psychological responses, behaviours and accomplishments that occur before, during, and after use [31]. In both Finland [17] and China [15,16], the trials have indicated positive user experiences for the tested mobile memory games.

In Finland, among the elderly players, the games concerned were seen as interesting, exciting, entertaining, and evidently engaged the participants' interest. The games were warmly welcomed and participants supported them as potential rehabilitation and recreation tools. The detailed results from the Finnish trial can be read in the study by Merilampi et al. [17], whereas the unpublished Chinese results are discussed below.

In China, the game was immediately accepted and all participants were able to understand the logic of each game. The players were asked to grade how much they felt playing these games would benefit memory training, dexterity, coordination, breaking routines and social interaction in their lives on a scale of $1-5$, where 1 is "very beneficial" and 5 is "inadequate". These results are presented in Figure 3. Based on the results, these games were seen to benefit memory training, in addition to dexterity, coordination and breaking routines. On the other hand, the effects on social interaction were not seen to be very beneficial. One player did not wish to comment the effects on social interaction.

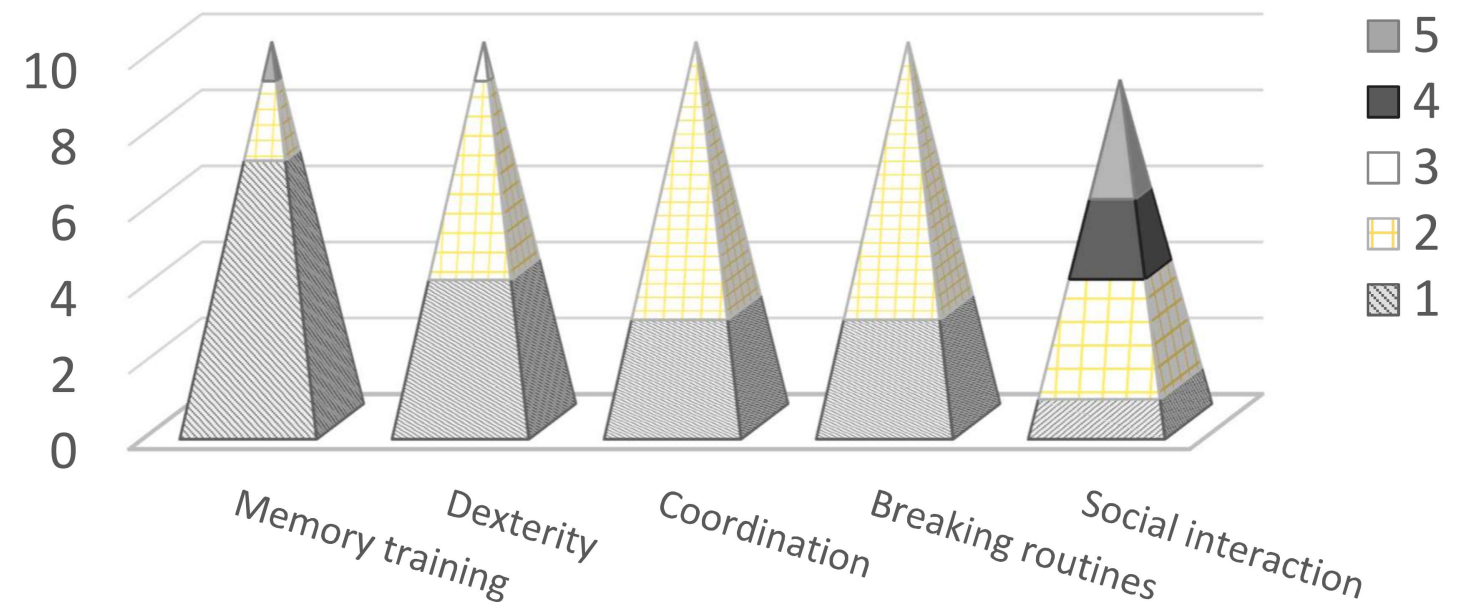

Figure 3. Results from the participant interviews in China (10 elderly players): The benefits of mobile gaming on different areas of their lives on a scale of $1-5$, where 1 is "very beneficial" and 5 is "inadequate".

The Finnish nursing staff feedback was collected via open comments. Overall, the feedback was extremely positive. Gaming tended to break routines and increase social interaction. The participants easily accepted and learned how to play the games, despite the rather high age of the players. The gaming was generally seen to appeal to the elderly and improvement in their gaming could be seen during the trial [17].

In China, the nursing staff were also interviewed following the gaming trial. The main challenge identified was that the elderly required help in playing the games, which meant that nursing staff would need to be present and able to help. This may require some extra training for the nursing staff. Out of the six interviewed nursing staff members, all found the games created a positive experience. Furthermore, all but one nurse found the games suitable for recreation and rehabilitation. They also stated that they would use such games in their own work, if suitable games were available. One nurse 
was worried about the effects of gaming on eyesight, but still said that such games could be used if available. The nursing staff were also asked to grade how they felt playing these games would benefit memory training, dexterity, coordination, breaking routines and social interaction on a scale of $1-5$, where 1 is "very beneficial" and 5 is "inadequate". These results are presented in Figure 4 . As can be seen, the results are very similar to those received from the players. In general, the nursing staff had a slightly more positive feeling about the effects of gaming. In particular, the nursing staff saw these games as beneficial for memory training, whereas the social effects of the games were not seen as beneficial. This feedback was encouraging in general; however, based on the experiences in organizing the trial, the positive attitudes are not enough. Service designing is required to support implementation of the games in elderly care practice. To summarise, after longer term trials the user experiences remained positive and games were seen to have both rehabilitative and recreational value.

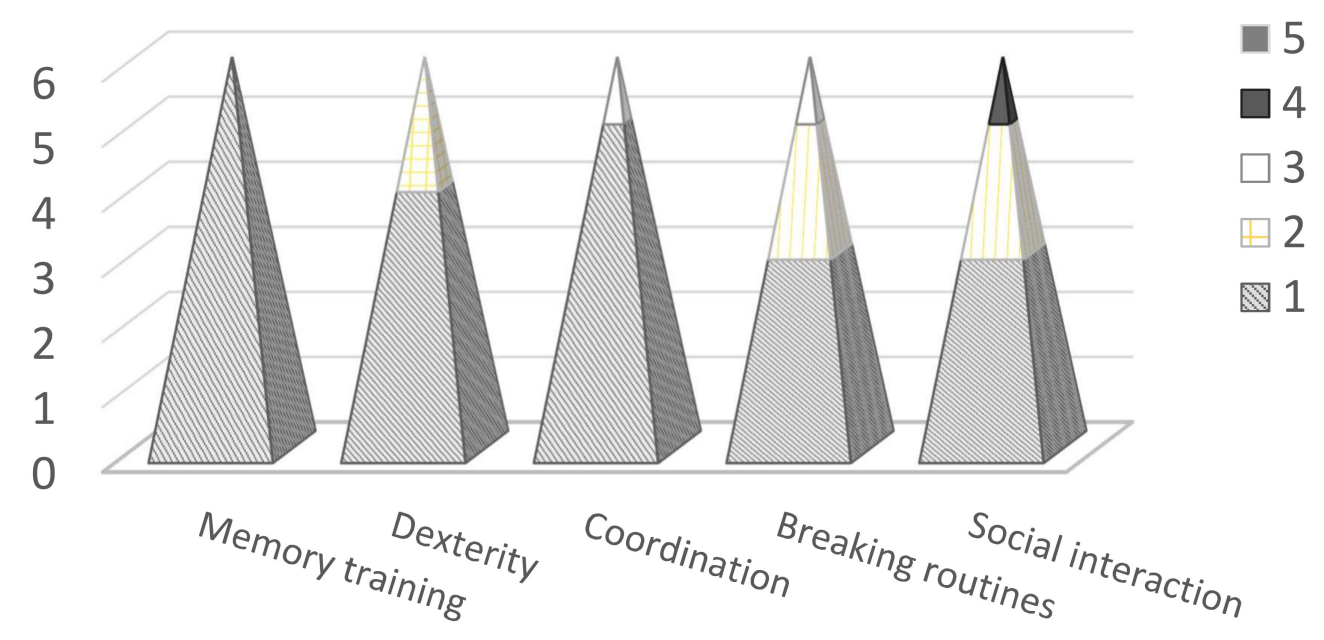

Figure 4. Results from the nursing staff interview in China (six staff members): The benefits of mobile gaming on different areas of the lives of the elderly on a scale of $1-5$, where 1 is "very beneficial" and 5 is "inadequate".

\subsection{Gaming Scores}

In this section, gaming scores and gaming activity are discussed, and results from the automatic data collection are provided to further investigate the effect of the games. Firstly, the Cat vs. Mouse game scores are discussed. Figure 5 presents the data from the Chinese trial. Overall, the Chinese game results were improved over time, the regression line equation being $0.0199 x+113.33$. In Finland the Cat vs. Mouse game scores also indicated improvement, the regression line equation being $0.4998 x-20.626$ [17]. It was concluded that three months of daily playing in Finland resulted in improved games scores compared to the Chinese participants. However, slight improvement was also seen in the trial with weekly frequency in China. It should be noted that Chinese players were slightly younger (with an average age of 84 , whereas the average age in the Finland study was 90) and game scores were already higher in the beginning; average game scores during the first day were 95.6 (China) and 48.2 (Finland), which may indicate better cognitive skills or better skills in using touch screen devices. However, in Finland the games and the tablet were described as easy to use without any major problems, which implies that technical skills did not crucially affect the result. The total number of playing times was larger in China compared to Finland, despite the different setting: 905 times in China and 726 in Finland. 


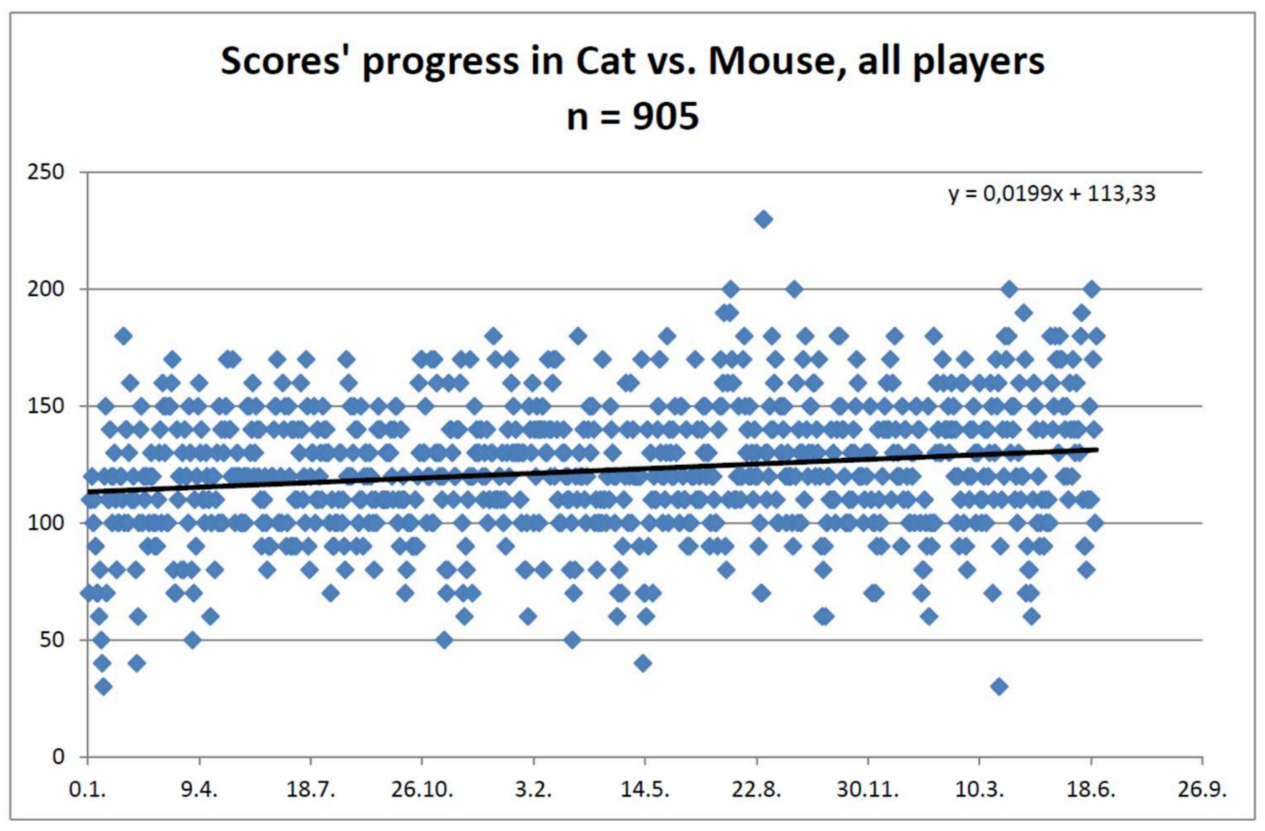

Figure 5. Cat vs. Mouse game scores in China ( $y$-axis illustrates game score and $x$-axis the dates).

The TMT game was played a total of 696 times in Finland and 1102 times in China. In the Finnish trial [17], a negative slope was commonly found in the TMT scores indicating improved game score (less time required to finish the game) at lower levels. Most of the players improved their TMT game scores between levels 10 to 20; however, after increasing the difficulty level up to 25 digits or more, the game times increased and improvement in the results ceased. The average slopes of game scores in China are given in Table 1.

Table 1. Gaming on different levels in TMT (in China).

\begin{tabular}{ccc}
\hline (Number of Digits) & Slope (All Results) & Number of Games Played \\
\hline 5 & 0.0537 & 84 \\
10 & 0.1634 & 54 \\
15 & -0.1579 & 95 \\
20 & -0.3489 & 171 \\
25 & 0.1615 & 158 \\
30 & -0.3159 & 184 \\
35 & -0.3168 & 141 \\
40 & -0.3973 & 215 \\
\hline
\end{tabular}

As seen from the results, the lower levels (5-15) were not played as much as the higher levels. Level 40 was played the most. This further supports the theory that the Chinese group possessed better cognitive skills, since in Finland only a couple of results were recorded at level 40 . The slopes in the Chinese trial are also commonly negative, indicating improved game scores. This implies that weekly gaming is effective when it comes to improvement in game scores. However, the changes might be different should the frequency vary. This requires further research. Since the aim of the paper was to justify the gaming service and its suitability, no deeper analysis of the game scores were performed at this point. The authors understand that the results are preliminary and further testing must be performed to analyse the health effects of the games and gaming services.

\subsection{Gaming Service Models}

The games which were used as a technology tool in this case study are mobile games built on the Android platform. Typically, mobile games are delivered through mobile stores. In our case the 
user group was elderly people and this distribution channel was not optimal for providing technology to this demographic. No matter how beneficial the game would be, the benefits cannot be realised if the game does not reach the target group. In addition, most people buy games for the enjoyment of gaming, whereas in our case the elderly would potentially be more interested in memory training. These view-points encouraged the development of alternative methods for providing the games to elderly individuals. Further, we should provide value-added services to the elderly, such as memory training/rehabilitation services, instead of just "selling" a game. The game could be used as a tool in this service. This thinking combined with the piloting experience for the two memory games in Finland and in China led to the development of two novel service models.

Service Model 1 (presented in Figure 6) is based on the Finnish trial, in which the gaming was managed by nursing personnel. In all customer journeys, it is essential to make it easy for the potential customer to find the service or the service provider. In our case, instead of relying on a mobile store, other marketing channels should be considered (newspaper commercials, visits to senior exhibitions and events, marketing to care homes, municipalities and relatives, direct phone calls, visits etc.). The actual service provided is much larger than the gameplay itself. The service could start from memory testing (similar to the trial in Finland), which is performed by a professional "owning the process". Based on the memory test results, an individual game plan can be made together with the client, taking into account their goals, characteristics and life situation (client involvement). The plan is followed by user training, in which an elderly person is taught to use the tablet as well as the game. This is performed in collaboration with the professional, to lower the threshold to begin using the tool and reduce user frustration. After user training, the client should follow the game plan at home. There are many ways of providing the game to the user, such as renting a tablet with the game, or installing the game on the person's own device. Support services should also be provided in case of problems in home gaming. Services could also include checkpoints, in which the user's gaming activity and progress is analysed (checking whether the client has followed the plan, and that the plan has been effective). After the checkpoint the game plan may be modified, if the results are not satisfactory. Otherwise the client can continue with the original plan. After the gaming period, new memory tests may be performed and combined with the data produced by the game to help determine the next steps. These steps may be, for example, to end the service, or start a new game service (with different games or different gaming sequences). If ending the service, the service provider could send offers and reminders to encourage new services at a later date.

The role of the game in the service is important as the game provides data about the progress, creates motivation, and can be used flexibly (as it is not dependent on a time or place). Entertainment and the ability to observe progress play important roles in creating and maintaining the player's motivation. In addition, the gaming data is important to the professionals, who can use the data to support decisions about the player's memory condition, as well as in evaluating the game plan and other related service needs.

Service Model 2 was based on the Chinese trial, in which the gaming service was provided by an external expert (due to unfamiliarity of the service within Chinese care culture, to avoid adding new responsibilities for care personnel). The gaming service, support services, and the game devices were all provided and managed by an external person, who delivered the service on a weekly basis to the elderly care home. This led to Service Model 2 (shown in Figure 7). The external service provider should offer services in the form of packages, to allow use by various customers. The services might include memory testing, game plan design, checkpoints and evaluations. Alternatively, the service may only include the gaming service (i.e., the devices and facilitator). The gaming service could be provided to elderly care homes, home care, private clients, elderly day-care providers etc. In addition, one service package could be a consultation service for game developers. This consultation service might include information about elderly users, usability, accessibility and help evaluating and piloting the game. On the other hand, the consultation might be targeted towards social and health care 
providers. Thus, the content revolves around "technology knowledge transfer" (what is possible with technology) and finding partners to develop need-based games.

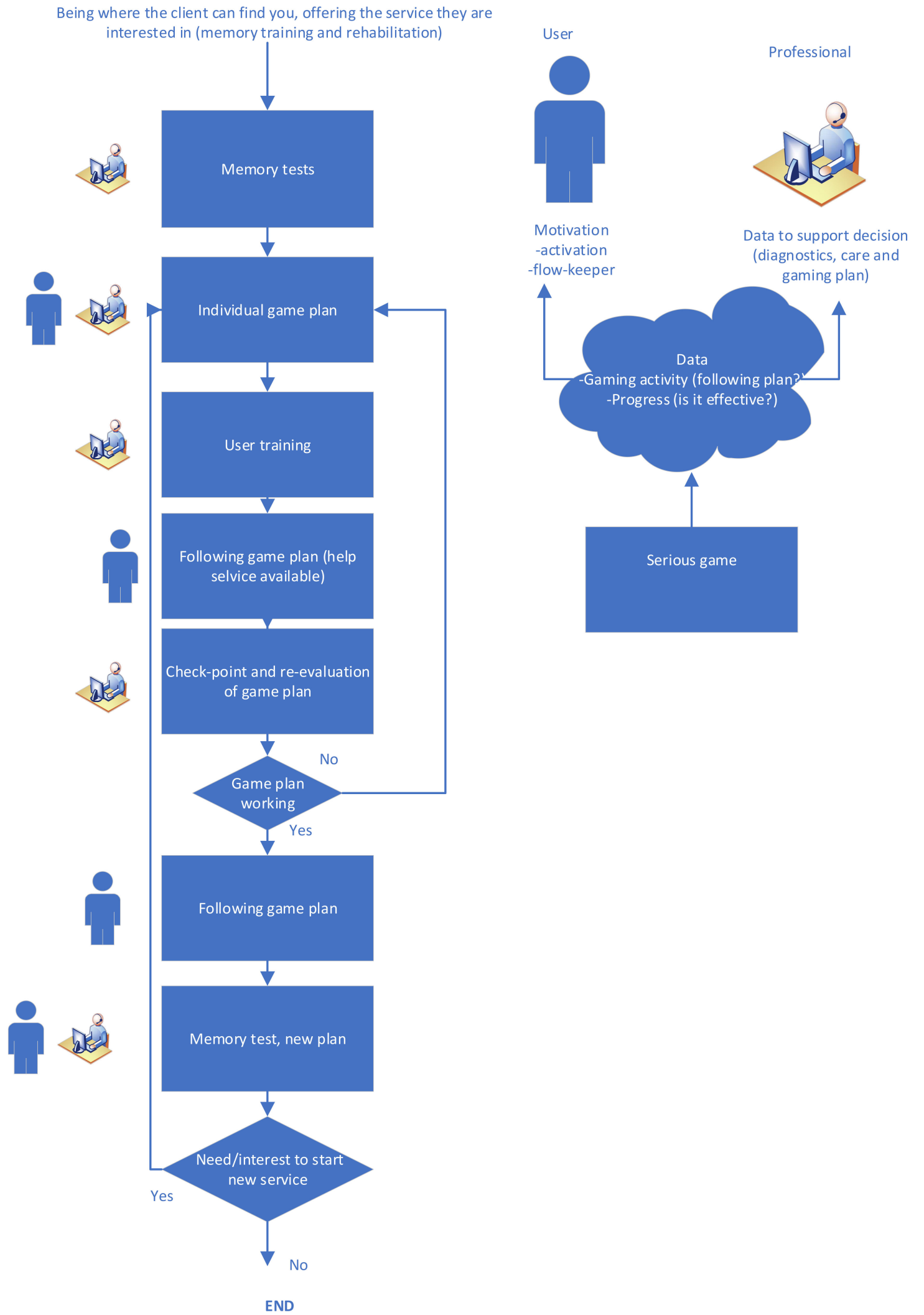

Figure 6. Service Model 1, based on the Finnish trial. 


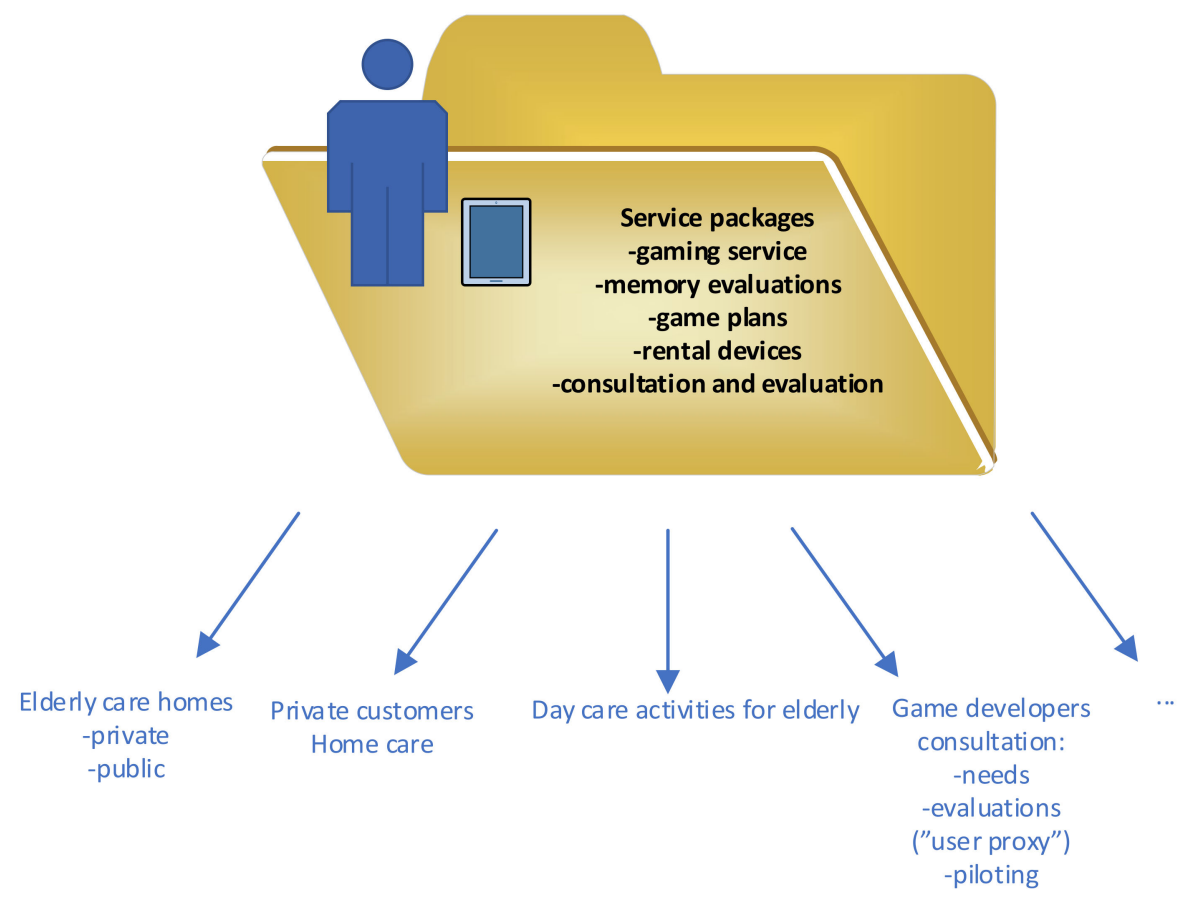

Figure 7. Service Model 2, based on the Chinese trial.

Due to the recreational value, games could also be implemented in a less professional manner. In the game trials, some elderly participants were found to take the lead in the gaming trial, helping and motivating others to play. In these cases, the gaming sessions could be organised amongst the elderly, without the need for constant professional guidance. This could create a feeling of meaningfulness for the managing elderly, in addition to recreational value for the participants.

The cultural characteristics are clearly seen in the service models. These culture-specific characteristics should be considered while developing products and services in different countries. To conclude, in the case of elderly users, serious games could benefit from the incorporation of support services (user training, help desk service, etc.), however more attention should be paid to value-added services, which are actually care services (memory tests, game plan, progress evaluation, etc.). This thought process would be beneficial to many other welfare technology products. In welfare technology contexts, one should think about:

- How would the technology solutions become a service?

- How would the solution become accessible and user-centric?

- What kinds of services are needed to implement the technology?

- What actually provides the most value for the customer?

- Are there any other application possibilities or user groups for the technology?

- Who could be the service provider for the customer?

These questions might help to focus on essential issues and design customer-oriented welfare technology services.

\section{Conclusions}

This paper studied the implementation of cognitively stimulating mobile gaming into elderly care. Two different mobile games were incorporated into the everyday lives of older adults in care homes in Finland and China. The game outcomes were automatically recorded and user feedback was collected from the participants and nursing staff. In both countries, the players and nursing staff found the games showed potential as self-managed rehabilitation tools. The biggest differences in the pilots involved the implementation process, and thus two novel service models for implementing mobile 
memory gaming into elderly care services were designed. In line with this, the proposed mobile games and their service models were identified as suitable means of implementation (i.e., delivered embedded into the care practices, or as a service independent of the care process). Studying the suitability of the developed service models for implementation of other serious games is the next step of our research.

Author Contributions: Conceptualization and methodology: S.M. and J.V.; software: A.K.; validation: N.P. and V.F.; formal analysis: M.L., S.M., A.K. and J.V.; investigation (literature review): A.P. and J.L.; data curation: M.L. and A.K.; writing—original draft preparation: S.M., A.K., A.P. and J.V.; writing—review and editing: N.P., V.F., L.J. and M.L.

Funding: This research received no external funding.

Conflicts of Interest: The authors declare no conflict of interest.

\section{References}

1. Serious Gaming for Future Seniors. Available online: https://stt.nl/stt/wp-content/uploads/2013/01/ STT_SG_ouderen_eng_HR-los.pdf (accessed on 5 February 2019).

2. Wiemeyer, J.; Kliem, A. Serious games in prevention and rehabilitation-A new panacea for elderly people? Eur. Rev. Aging Phys. Act. 2012, 9, 41-50. [CrossRef]

3. Nauta, H.; Spil, T.A.M. Change your lifestyle or your game is over: The design of a serious game for diabetes. In Proceedings of the IEEE 1st International Conference on Serious Games and Applications for Health (SeGAH), Braga, Portugal, 16-18 November 2011; pp. 1-7.

4. Ferreira, C. Gamification of stroke rehabilitation exercises using a smartphone. In Proceedings of the 8th International Conference on Pervasive Computing Technologies for Healthcare. ICST (Institute for Computer Sciences, Social-Informatics and Telecommunications Engineering), Oldenburg, Germany, 20-23 May 2014; pp. 282-285.

5. Martins, T.; Carvalho, V.; Soares, F. A serious game for rehabilitation of neurological disabilities: Premilinary study. In Proceedings of the IEEE 4th Portuguese Meeting on Bioengineering (ENBENG), Porto, Portugal, 26-28 February 2015; pp. 1-5.

6. Madeira, R.N.; Costa, L.; Postolache, O. PhysioMate-Pervasive physical rehabilitation based on NUI and gamification. In Proceedings of the International Conference and Exposition on Electrical and Power Engineering (EPE), Iasi, Romania, 16-18 October 2014; pp. 612-616.

7. Deponti, D.; Maggiorini, D.; Palazzi, C.E. Smartphone's physiatric serious game. In Proceedings of the IEEE 1st International Conference on Serious Games and Applications for Health (SeGAH), Braga, Portugal, 16-18 November 2011; pp. 1-8.

8. Miloff, A.; Marklund, A.; Carlbring, P. The challenger app for social anxiety disorder: New advances in mobile psychological treatment. Int. Interven. 2015, 2, 382-391. [CrossRef]

9. Martins, T.; Carvalho, V.; Soares, F.; Moreira, M.F. Serious game as a tool to intellectual disabilities therapy: Total challenge. In Proceedings of the IEEE 1st International Conference on Serious Games and Applications for Health (SeGAH), Braga, Portugal; 16-18 November 2011; pp. 1-7.

10. Chang, C.; Hinze, A.; Bowen, J.; Gilbert, L.; Starkey, N. Mymemory: A mobile memory assistant for people with traumatic brain injury. Int. J. Hum.-Comput. Stud. 2018, 117, 4-19. [CrossRef]

11. Hornos, M.J.; Rute-Pérez, S.; Rodríguez-Domínguez, C.; Rodríguez-Almendros, M.L.; Rodríguez-Fórtiz, M.J.; Caracuel, A. Visual Working Memory Training of the Elderly in VIRTRAEL Personalized Assistant. In Personal Assistants: Emerging Computational Technologies; Costa, A., Julian, V., Novais, P., Eds.; Intelligent Systems Reference Library; Springer: Cham, Switzerland, 2018; Volume 132.

12. Silva, B.M.; Rodrigues, J.J.; de la Torre Díez, I.; López-Coronado, M.; Saleem, K. Mobile-health: A review of current state in 2015. J. Biomed. Inform. 2015, 56, 265-272. [CrossRef] [PubMed]

13. Oh, H.; Rizo, C.; Enkin, M.; Jadad, A. What is eHealth (3): A systematic review of published definitions. J. Med. Inter. Res. 2005, 7, e1. [CrossRef] [PubMed]

14. Lanyu, X.; Heather, A.F.; Weisong, S. User centric design for aging population: Early experiences and lessons. In Proceedings of the IEEE Connected Health: Applications, Systems and Engineering Technologies (CHASE), Washington, DC, USA, 27-29 June 2016. 
15. Merilampi, S.; Koivisto, A.; Sirkka, A.; Xiao, X.; Min, Y.; Ye, L.; Chujun, X.; Chen, J.; Raumonen, P.; Virkki, J. The Cognitive Mobile Games for Older Adults-A Chinese User Experience Study. In Proceedings of the IEEE International Conference on Serious Games and Applications for Health (SEGAH), Perth, Australia, 2-4 April 2017.

16. Merilampi, S.; Koivisto, A.; Virkki, J. Activation Game for Older Adults-Development and Initial User Experiences. In Proceedings of the IEEE International Conference on Serious Games and Applications for Health (SEGAH), Vienna, Austria, 16-18 May 2018.

17. Merilampi, S.; Sirkka, A.; Leino, M.; Koivisto, A.; Finn, E. Cognitive mobile games for memory impaired older adults. J. Assist. Technol. 2014, 8, 207-223. [CrossRef]

18. Fairchild, J.K.; Scogin, F.R. Training to enhance Adult Memory (TEAM): An investigation of the effectiveness of a memory training program with older adults. Aging Mental Health 2010, 14, 364-373. [CrossRef] [PubMed]

19. McCough, E.L.; Kelly, V.E.; Logsdon, R.G.; McCurry, S.M.; Cochrane, B.B.; Engel, J.M.; Teri, L. Associations between physical performance and executive function in older adults with mild cognitive impairment: Gait speed and the timed "Up \& Go" test. Phys. Ther. 2011, 91, 1198-1210.

20. Szturm, T.; Betker, A.L.; Moussavi, Z.; Desai, A.; Goodman, V. Effects of an interactive computer game exercise regimen on balance impairment in frail community-dwelling older adults: A randomized controlled trial. Phys. Ther. 2011, 91, 1449-1462. [CrossRef] [PubMed]

21. Trail Making Test. Available online: http://apps.usd.edu/coglab/schieber/psyc423/pdf/IowaTrailMaking. pdf (accessed on 1 November 2018).

22. Lezak, M.D.; Howieson, D.B.; Loring, D.W. Neuropsychological Assessment, 4th ed.; Oxford University Press: New York, NY, USA, 2004.

23. Reitan, R.M. Validity of the Trail Making test as an indicator of organic brain damage. Percept Mot Skills 1958, 8, 271-276. [CrossRef]

24. Garrett, J.J. Elements of User Experience, the: User-Centered Design for the Web and Beyond; Pearson Education: New York, NY, USA, 2010.

25. Hwang, M.-Y.; Hong, J.-C.; Hao, Y.; Jong, J-T. Elders' usability, dependability, and flow experiences on embodied interactive video games. Educ. Gerontol. 2011, 37, 715-731. [CrossRef]

26. Snowden, M.; Steinman, L.; Mochan, K.; Grodstein, F.; Prohaska, T.R.; Thurman, D.J.; Brown, D.R.; Laditka, J.N.; Soares, J.; Zweiback, D.J.; et al. Effect of exercise on cognitive performance in community-dwelling older adults: Review of intervention trials and recommendations for public health practice and research. J. Am. Geriat. Soc. 2011, 59, 704-716. [CrossRef] [PubMed]

27. Vekara, J. International Business Negotiations between Finnish and Chinese Business People. Master's Thesis, University of Vaasa, Vaasa, Finland, 2014.

28. Purmonen, M. Influence of Cultural Differences on Finnish-Chinese Cooperation at UPM-Kymmene's three Asian Sales Offices. Master's Thesis, University of Jyväskylä, Jyväskylä, Finland, 2000.

29. King, P.C.; Zhang, W. The role of face in a Chinese context of trust and trust building. Int. J. Cross Cult. Manag. 2018, 18, 149-173. [CrossRef]

30. Näsi, M.J.; Räsänen, P.; Keipi, T.; Oksanen, A. Trust and victimization: A cross-national comparison of Finland, the U.S., Germany and UK. Res. Finnish Soc. 2017, 10, 119-131.

31. ISO 9241-210:2010. Ergonomics of Human-System Interaction-Part 210: Human-Centred Design for Interactive Systems. Available online: https://www.iso.org/obp/ui/\#iso:std:iso:9241:-210:ed-1:v1:en (accessed on 5 February 2019).

(C) 2019 by the authors. Licensee MDPI, Basel, Switzerland. This article is an open access article distributed under the terms and conditions of the Creative Commons Attribution (CC BY) license (http://creativecommons.org/licenses/by/4.0/). 